\title{
Magnetopause energy and mass transfer: results from a global MHD simulation
}

\author{
M. Palmroth, T. V. Laitinen, and T. I. Pulkkinen \\ Finnish Meteorological Institute, Space Research Division, Helsinki, Finland
}

Received: 28 June 2006 - Revised: 23 October 2006 - Accepted: 8 November 2006 - Published: 21 December 2006

\begin{abstract}
We use the global MHD model GUMICS-4 to investigate the energy and mass transfer through the magnetopause and towards the closed magnetic field as a response to the interplanetary magnetic field (IMF) clock angle $\theta=\arctan \left(B_{Y} / B_{Z}\right)$, IMF magnitude, and solar wind dynamic pressure. We find that the mass and energy transfer at the magnetopause are different both in spatial characteristics and in response to changes in the solar wind parameters. The energy transfer follows best the $\sin ^{2}(\theta / 2)$ dependence, although there is more energy transfer after large energy input, and the reconnection line follows the IMF rotation with a delay. There is no clear clock angle dependence in the net mass transfer through the magnetopause, but the mass transfer through the dayside magnetopause and towards the closed field occurs preferably for northward IMF. The energy transfer occurs through areas at the magnetopause that are perpendicular to the subsolar reconnection line. In contrast, the mass transfer occurs consistently along the reconnection line, both through the magnetopause and towards the closed field. Both the energy and mass transfer are enhanced in response to increased solar wind dynamic pressure, while increasing the IMF magnitude does not affect the transfer quantities as much.
\end{abstract}

Keywords. Magnetospheric physics (Magnetopause, cusp, and boundary layers; Solar wind-magnetosphere interactions) - Space plasma physics (Numerical simulation studies)

\section{Introduction}

Understanding and quantifying the energy and mass transfer from the solar wind to the magnetosphere has been one of the the important long-standing question in space physics.

Correspondence to: M. Palmroth

(minna.palmroth@fmi.fi)
While observationally the question is difficult to solve globally, the behavior of the various activity indices (such as the Dst and AE) have suggested that the southward orientation of the interplanetary magnetic field (IMF) has a dominant role in the energy transfer (e.g., Burton et al., 1975; Akasofu, 1981). This has been explained by reconnection transferring energy from the solar wind, as first suggested by Dungey (1961). While reconnection between the IMF and terrestrial magnetic field occurs practically for any orientation of the IMF, during southward IMF a large portion of the subsolar magnetopause is opened along the reconnection line, while for northward IMF reconnection moves to the lobes tailward of the cusps (e.g., Luhmann et al., 1984). Deducing from the polar cap potential difference, which can be thought of a proxy for the reconnection efficiency, the energy coupling between the solar wind and the magnetosphere during northward IMF constitutes up to a few tens of percent of that during southward IMF (e.g., Shepherd et al., 2002).

As reconnection allows also mass transfer from one plasma system to another along the interconnected field lines, one could imagine that the times of southward IMF would also signify efficient mass transfer between the solar wind and the magnetosphere. However, the plasma population inside the plasma sheet becomes dense and cool near the flanks adjacent to the magnetosheath during northward IMF (Wing and Newell, 2002; Tsyganenko and Mukai, 2003), implying efficient plasma entry from the solar wind. Hence, at least lobe reconnection (behind the cusps) and KelvinHelmholtz instability acting favorably for northward IMF have been suggested to control the mass transfer between the solar wind and the magnetosphere (e.g., Li et al., 2005; Nykyri and Otto, 2001; Hasegawa et al., 2004). Also diffusion through the magnetopause may come into play (Johnson and Cheng, 1997); however, it is not clear whether the diffusion would particularly favor northward IMF conditions. Since the plasma sheet plasma may later occupy the ring current (Thomsen et al., 2003) having a crucial role in magnetic

Published by Copernicus GmbH on behalf of the European Geosciences Union. 
storms, processes involved in populating the plasma sheet are of major interest also from the practical space weather point of view.

Besides the IMF dependence, other solar wind parameters influencing the mass and energy transfer have also been investigated. Hubert et al. (2006) show that the compression of the magnetosphere due to a dynamic pressure pulse drives flux closure in the tail, and hence the energy transfer may also be dependent on the dynamic pressure. Furthermore, Lu et al. (2004) present evidence that compressional waves after a dynamic pressure impulse may modulate reconnection and lead to ion injections into the magnetosphere. Still, the proxy used most often for the energy transfer, the $\epsilon=4 \pi \mu_{0}^{-1} l_{0}^{2} v B^{2} \sin ^{4}(\theta / 2)$ parameter (Akasofu, 1981), where $\theta=\arctan \left(B_{Y} / B_{Z}\right)$ is the IMF clock angle, and $l_{0}$ is a scaling parameter, depends on the IMF magnitude $B$, but the solar wind dynamic pressure $p_{\mathrm{dyn}}=\rho v^{2}$ is present only through the solar wind speed $v$, while the solar wind density $\rho$ is absent.

Currently, the global magnetohydrodynamic (MHD) codes provide the only means to self-consistently model the solar wind - magnetosphere - ionosphere system and to quantitatively study the spatial distribution of the global energy and mass transfer. Statistical studies on the filling of the plasma sheet report larger densities near the flanks (Wing and Newell, 2002), while it is not clear whether the mass transfer occurs at the flanks. Namely, using a test particle approach utilizing the electromagnetic fields from a global MHD simulation, Li et al. (2005) reported that lobe reconnection during northward IMF captures magnetosheath plasma, which then sinks into the plasma sheet while convecting tailward. Using another global MHD code, Palmroth et al. (2003) found that electromagnetic energy focusses towards the magnetopause controlling the energy transfer both spatially and temporally, while they did not investigate the spatial extent of mass transfer. The reconnection efficiency in the tail and at the magnetopause have also been quantified using a global MHD code (Laitinen et al., 2005, 2006). The tail reconnection was found to process half of the incoming magnetopause energy, indicating that the tail reconnection in the global MHD code is an efficient process and central to the tail dynamics. This implies that the tendency to slow reconnection rates in the MHD formulation (Birn et al., 2001) does not necessarily mean that reconnection could not realistically operate in global MHD simulations.

At present, the global MHD simulations cannot answer the major unsolved question of the relative impact between different energy and mass transfer mechanisms, as the usual spatial resolution $\left(\sim 0.2 R_{E}\right)$ is not enough to model KelvinHelmholtz vortices at the magnetopause. Furthermore, a property of ideal MHD is that all boundaries get thinner when increasing the grid resolution. Hence, the diffusion coefficient depending on the spatial gradient of the magnetopause would be unreliable and a function of the grid spacing, making the assessment of the diffused population through the magnetopause ambiguous. However, as previously mentioned, processes related to reconnection can be studied using the global MHD (Laitinen et al., 2005, 2006), even if the reconnection appears as a consequence of the numerical solution of the ideal MHD equations.

In this paper, we continue the work of Palmroth et al. (2003) to fully characterize the energy transfer from the solar wind into the magnetosphere, and for the first time attempt to quantify the mass transfer through the magnetopause and towards the closed field using a global MHD simulation. As a new feature, we investigate the dependence of the energy and mass transfer on the IMF and solar wind dynamic pressure. We study these dependencies using synthetic runs with controlled solar wind input parameters. The initial results from these runs were published in Palmroth et al. (2006). The paper is organized as follows: First we introduce the global MHD code as a tool to study the near-Earth space, as well as briefly summarize the developed methods with which the energy and mass transfer may be investigated. Second, we present our results of the energy transfer as functions of the IMF clock angle $\theta$, IMF magnitude, and solar wind dynamic pressure. We then characterize the mass transfer, both through the magnetopause as well as through the surface formed by the last closed field lines - again as functions of $\theta$, IMF magnitude, and solar wind dynamic pressure. Finally, we end the paper with our discussion and conclusions.

\section{Model and methods}

\subsection{GUMICS-4 global MHD code}

GUMICS-4 (Janhunen, 1996) is a computer code designed for solving the coupled solar wind - magnetosphere - ionosphere system. The solar wind and magnetosphere are modeled by solving the fully conservative MHD equations in a simulation box extending from $32 R_{E}$ to $-224 R_{E}$ in the $X_{\mathrm{GSE}}$ direction and $\pm 64 R_{E}$ in $Y_{\mathrm{GSE}}$ and $Z_{\mathrm{GSE}}$. Near the Earth the MHD domain reaches a spherical shell with a radius of $3.7 R_{E}$. The ionosphere is electrostatic and uses the electron precipitation and field-aligned currents from the magnetosphere to solve the ionospheric potential, which is mapped back to the magnetosphere and used there as a boundary condition. The grid in the MHD simulation box is a Cartesian octogrid, and it is adaptive allowing finer grid to be used when the spatial gradients become large. This ensures that the magnetopause is a sharp boundary, whose properties are well in accordance with empirical models based on large statistical surveys (Palmroth et al., 2001). Solar wind density $n$, temperature $T$, velocity $\boldsymbol{v}$ and magnetic field $\boldsymbol{B}$ are treated as boundary conditions on the sunward wall of the simulation box; outflow conditions are applied on the other walls of the simulation box. 


\subsection{Determining energy transfer through magnetopause}

Palmroth et al. (2003) introduced a method determining the energy transfer through the magnetopause using GUMICS4. The method detects the magnetopause surface for each output file (usually saved once in $5 \mathrm{~min}$ ) and computes the transferred energy through the surface using the simulation results. The method determines the magnetopause by finding approximately the inner edge of the void encompassed by the solar wind stream lines. The stream line surface is found to coincide with the spatial gradients existing at the magnetopause; however, it is considerably smoother than a surface based on, e.g., plasma or current density. Hence, the stream line surface does not contain significant bulges or valleys that would deteriorate the surface orientation (having significance in the actual energy transfer calculation).

Once the surface is detected, the total transferred energy through the magnetopause, $P_{M P}$, is defined as

$P_{M P}=\int d A \boldsymbol{K} \cdot \hat{\boldsymbol{n}}$,

where $d A$ is the area of the surface element and $\hat{\boldsymbol{n}}$ is the unit vector perpendicular to the surface element pointing outwards from the magnetopause. $\boldsymbol{K}$ is the total energy flux, and is defined as

$\boldsymbol{K}=\left(U+P-\frac{B^{2}}{2 \mu_{0}}\right) \boldsymbol{v}+\frac{1}{\mu_{0}} \boldsymbol{E} \times \boldsymbol{B}$,

where $P$ is the pressure, $\boldsymbol{B}$ is the magnetic field, $\boldsymbol{v}$ is velocity of solar wind, $\boldsymbol{E}=\boldsymbol{B} \times \boldsymbol{v}$ is the electric field, and $U=P /(\gamma-1)+\rho v^{2} / 2+B^{2} / 2 \mu_{0}$ is the total energy density. Due to the sign convention of the surface normal, negative values indicate transfer towards the surface, and vice versa. For obtaining the total energy through the surface the integration in Eq. (1) is carried out from the nose of the magnetopause to $-30 R_{E}$ in the tail over all azimuthal directions in the $Y Z$ plane.

\subsection{Determining the reconnection site location}

Traditionally, reconnection is identified from the observational data through its characteristic plasma and magnetic field signatures (e.g., Øieroset et al., 2004). Basically, breaking of the frozen-in condition requires a parallel electric field, which, however, is identically zero in ideal MHD. Therefore, other characteristics of reconnection must be used when tracking its location in a global MHD simulation. As observed in any 2-dimensional reconnection settings, such as the classic Sweet-Parker reconnection (Sweet, 1958; Parker, 1957), the diffusion region contains four separate segments of field lines. In the magnetospheric context they may be identified as closed, open, towards and away from the Earth. Based on this property, Laitinen et al. (2006) introduced a general method to locate the separator line in a threedimensional grid. The method searches for locations where the four types of field lines meet in a spatially limited region. Laitinen et al. (2006) conclude that the method, named the "four-field junction" (FFJ) condition, is robust and in agreement with other characteristics of reconnection, such as the sign change of the normal component of the magnetic field in the tail current sheet. Laitinen et al. (2006) emphasize that the FFJ condition alone is not sufficient for tracking the reconnection sites, but may be used as a reconnection characterization if electromagnetic energy is converted into other forms of energy at the FFJ location. In GUMICS-4, this occurs at the dayside magnetopause approximately for clock angles between $60^{\circ}<\theta<300^{\circ}$, and behind the cusps otherwise (not shown).

\section{Characteristics of transfer quantities}

Figure 1 and Table 1 present the solar wind input for the runs presented in this paper. The IMF clock angle (Fig. 1a) rotates from $0^{\circ}$ to $360^{\circ}$ with $10^{\circ}$ steps such that each clock angle value is kept constant for $10 \mathrm{~min}$. Altogether, the full IMF rotation takes $6 \mathrm{~h}$ in all four runs. Figures $1 \mathrm{~b}$ and $1 \mathrm{c}$ present the IMF y- and z-components, respectively, computed using the clock angle with IMF magnitude $5 \mathrm{nT}$ (solid) and $10 \mathrm{nT}$ (dashed). As the clock angle was the only variable that changed during the course of the runs, four runs were needed to investigate the influence of the two values of IMF magnitude and solar wind dynamic pressure $p_{d y n}$. As can be seen in Table 1, both the solar wind x-directed velocity $v_{x}$ and density $n$ were changed in order to vary the dynamic pressure, because these variables usually change in concert in the solar wind. Table 1 further shows that IMF x-component as well as the $y$ - and z-components of solar wind velocity, and the dipole tilt angle are zero in all runs. The runs were initiated by running IMF $(\mathrm{x}, \mathrm{y}, \mathrm{z})=(0,2,5) \mathrm{nT}$ for one hour before the full rotation started.

\subsection{Energy transfer at the magnetopause}

Panels (a-f) of Fig. 2 present the instantaneous distributions of energy transfer for Run \#1, integrated from the nose of the magnetopause to $-30 R_{E}$ in the tail. Each sector shows the sum of transferred energy taking place in the angular direction shown in the outer circle, viewing from the Sun looking tailward. The size of the sector is normalized to the outer circle $(800 \mathrm{GW})$. The IMF clock angle at the time for which the distribution is plotted is indicated by a red arrow. Blue color indicates net energy flow towards the magnetopause, while the black circles plotted over the sectors show the location where the FFJ condition holds, i.e., where reconnection is likely to occur. For the energy transfer there is no information on the $X$-distance at which the energy transfer occurs, although we have previously shown that the energy transfer occurs predominantly Sunward of the distance $X=-10 R_{E}$ (Palmroth et al., 2003). However, the black circles range 
Table 1. Parameters for synthetic runs.

\begin{tabular}{lccccc}
\hline Run \# & $|\mathrm{IMF}|[\mathrm{nT}]$ & $\mathrm{p}_{\text {dyn }}[n P a]$ & $\mathrm{v}_{x}[\mathrm{~km} / \mathrm{s}]$ & $\mathrm{n}[\# / \mathrm{cc}]$ & $\mathrm{v}_{y, z}[\mathrm{~km} / \mathrm{s}], \mathrm{B}_{x}[\mathrm{nT}]$, tilt $\left[{ }^{\circ}\right]$ \\
\hline 1 & 5 & 2 & -400 & 7.3 & 0 \\
2 & 5 & 8 & -600 & 13.3 & 0 \\
3 & 10 & 2 & -400 & 7.3 & 0 \\
4 & 10 & 8 & -600 & 13.3 & 0 \\
\hline
\end{tabular}

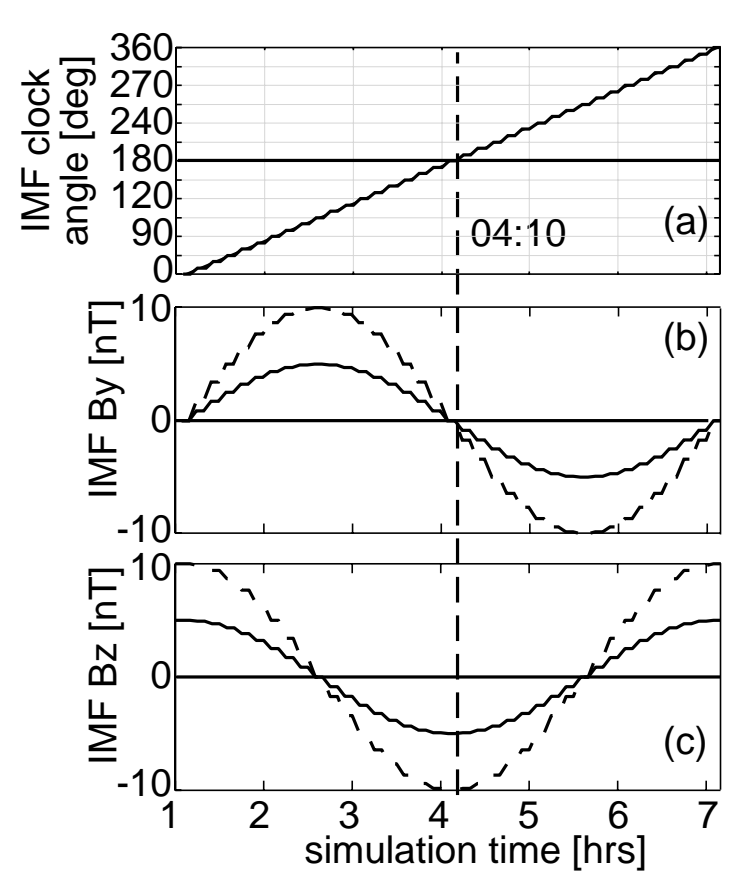

Fig. 1. Time evolution of (a) IMF clock angle, (b) IMF $y$ component, and (c) IMF z-component for simulation runs; solid (dashed) line is for runs where IMF magnitude is 5 (10) nT. See Table 1 for further details of the run parameters.

from the nose of the magnetopause (center of the panel) to the dawn-dusk terminator, which has been chosen as the limit of the FFJ. This is because only on the dayside magnetopause the FFJ is accompanied by considerable electromagnetic energy annihilation, and can thus be considered to mark the reconnection line (Laitinen et al., 2006).

Figure $2 \mathrm{~g}$ shows the total integrated energy across the entire magnetopause (solid, Eq. 1), and scaled $\sin ^{2}(\theta / 2)$ (dashed), both as a function of the clock angle (and time), in Run\#1. The vertical dashed lines indicate the times for which the instantaneous energy transfer distributions are shown above.

During $\theta=0^{\circ}$ (Fig. 2a), the energy transfer is at minimum and occurs at dawn (dusk) high-latitudes in the Northern (Southern) Hemisphere. For clock angles between $300^{\circ}$ and $60^{\circ}$ the examination of the electromagnetic energy annihilation indicates that reconnection occurs only at small locations behind the cusps (not shown), while the FFJ method often shows reconnection at low latitudes. This is because lobe reconnection generates open field lines that convect sunward to the other hemisphere, which causes the method to find all four types of field lines at the dayside low latitudes. Although misleading, these locations usually do not form a clear "reconnection line" but appear to lie on specific field lines (as is the case in Fig. 2a), and therefore their existence may be regarded as evidence of lobe reconnection and sunward convection. Nevertheless, it is clear that even for due north IMF, the (small) energy transfer occurs away from the reconnection location, which in this case occurs at dusk (dawn) high latitudes in the Northern (Southern) Hemisphere. The reconnection location is away from noon meridian at $\theta=0^{\circ}$ probably because the run was initialized with finite positive IMF y-component, which still shows before the IMF rotation is clearly in action.

In Fig. 2b, the IMF has rotated for over an hour to $\theta=60^{\circ}$. The energy transfer is enhanced, and it occurs clearly at dawn (dusk) high-latitudes in the Northern (Southern) Hemisphere. The reconnection occurring in the dusk (dawn) sectors in the Northern (Southern) Hemisphere already has a low-latitude component, although it does not yet reach the very nose of the magnetopause. Furthermore, the sunward convection due to behind-cusp reconnection has ceased, as can be seen from the fact that the FFJ locations now form a clear (discontinuous) "reconnection line". In fact, as reconnection now has the low-latitude component, the open field lines convect to the nightside through dawn (dusk) high latitudes in the Northern (Southern) Hemisphere, as can be seen from the schematic Fig. 3a adopted from Cowley et al. (1991). On the other hand, Fig. 3c shows that where-ever a field line moves with a finite angle with the magnetopause, geometry between the magnetosheath bulk flow and the moving field line demands that the Poynting vector $S$ points towards the magnetopause. This implies that electromagnetic energy, which forms the largest component of the transferring energy in all runs at all times, focusses toward the magnetopause at locations where field lines convect to nightside. This also explains why the energy is mainly transferred sunward of $X=-10 R_{E}$ (Palmroth et al., 2003), because tailward of this distance the field lines are already more aligned with the magnetosheath bulk flow, making the Poynting vector towards the magnetopause small. 


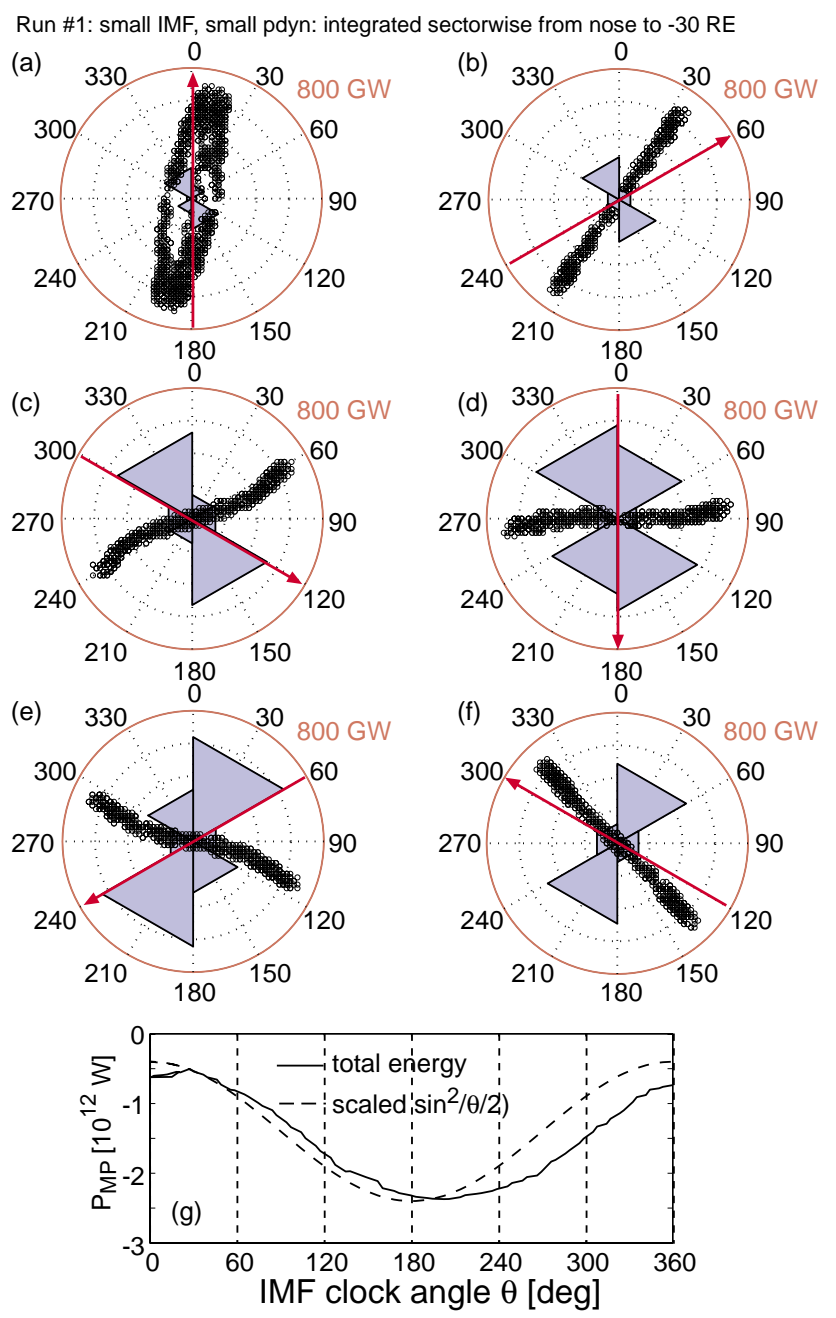

Fig. 2. (a-f) Instantaneous distributions of azimuthal magnetopause energy transfer at angles indicated by dashed vertical lines in panel $(\mathrm{g})$, which presents the time evolution of total transferred energy for Run \#1. Blue color indicates inward (towards magnetopause) energy to sectors shown outside the outer circle. The sectors scale from $0 \mathrm{GW}$ at the centre to $800 \mathrm{GW}$ at the outer circle. The IMF clock angle direction is indicated by a red arrow, while black circles show the locations where the "four-field junction" condition holds; i.e., where reconnection is likely to occur (note that the dayside black circles in panel (a) indicate sunward convection, not reconnection, which occurs behind the cusps as deduced from the annihilation of magnetic energy). (g) The solid line is the total transferred energy as function of clock angle (and time), while the dashed line is $\sin ^{2}(\theta / 2)$. Although the figure is for Run \#1, the other runs behave qualitatively similarly, only the amount of transferred energy in the other runs is different (see Palmroth et al., 2006).

In Fig. 2c energy is still transferred in the dawn (dusk) sector in the Northern (Southern) high latitudes, perpendicular to the reconnection line. As the low latitude reconnection has now fully begun, the high latitude convection and
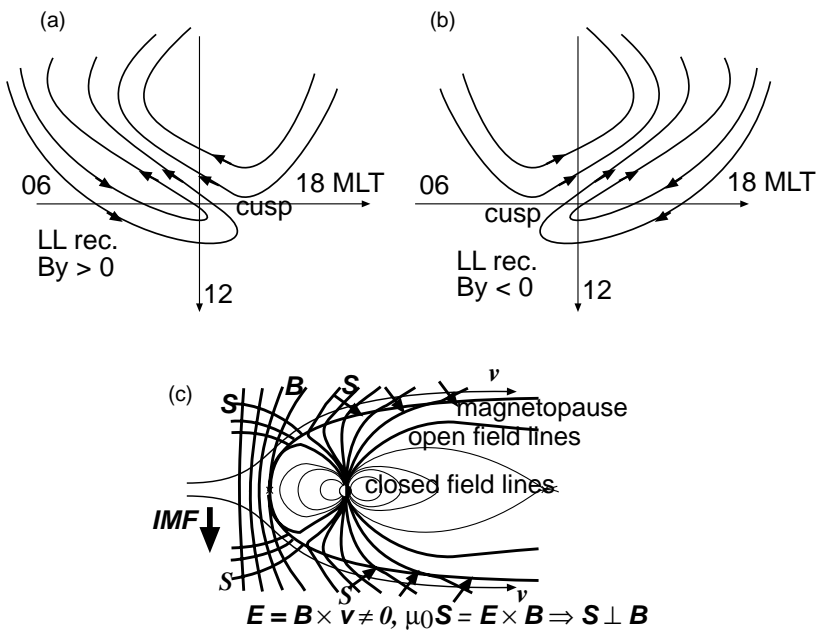

Fig. 3. (a) High latitude convection viewed above the Northern Hemisphere, if IMF $y$ is positive, and low-latitude (LL) reconnection is taking place. The convection is towards afternoon sector in the Southern Hemisphere (not shown). (b) Same as panel (a) but for negative IMF $y$; the convection is toward dawn sector in the Southern Hemisphere. (a and b after Cowley et al., 1991). (c) Poynting flux focussing: if a field line traverses tailward with a finite angle with the magnetopause and magnetosheath flow, then geometry demands that Poynting vector $\boldsymbol{S}$ points toward the magnetopause and is perpendicular to the open field line (after Palmroth et al., 2003).

consequently the amount of energy over dawn (dusk) sector in Northern (Southern) Hemisphere has been enhanced. In Fig. 2d, the IMF is due southward, and the reconnection line lies near the equator and extends throughout the low latitudes. This implies that the field lines convect tailward throughout the high latitudes. Consequently, the energy transfer is enhanced in both high-latitude dusk and dawn sectors on both hemispheres.

In Fig. 2e, the clock angle has rotated to $240^{\circ}$. Figure $3 b$ shows that during low latitude reconnection and negative IMF y-component, the open field lines convect through dusk (dawn) sector on Northern (Southern) Hemisphere, which is where the largest energy transfer is also taking place due to Poynting flux focussing (Fig. 3c). Notice also that the dawn (dusk) high latitude sector in the Northern (Southern) Hemisphere show more enhanced energy transfer than dusk (dawn) sector during similar exterior conditions in Fig. 2c (except for sign of IMF $y$ ). Furthermore, the reconnection line is slightly more aligned with low latitudes than in Fig. 2c. Both facts imply, respectively, that 1) convection has not altogether ceased from dawn (dusk) sector in the North (South), and that 2) convection is more enhanced in dusk (dawn) high latitudes. Consequently, more energy is transferring during $\theta=240^{\circ}$ than during $\theta=120^{\circ}$, which shows also in Fig. $2 \mathrm{~g}$. Palmroth et al. (2006) have speculated that this might be due to hysteretic behavior of magnetopause reconnection. 


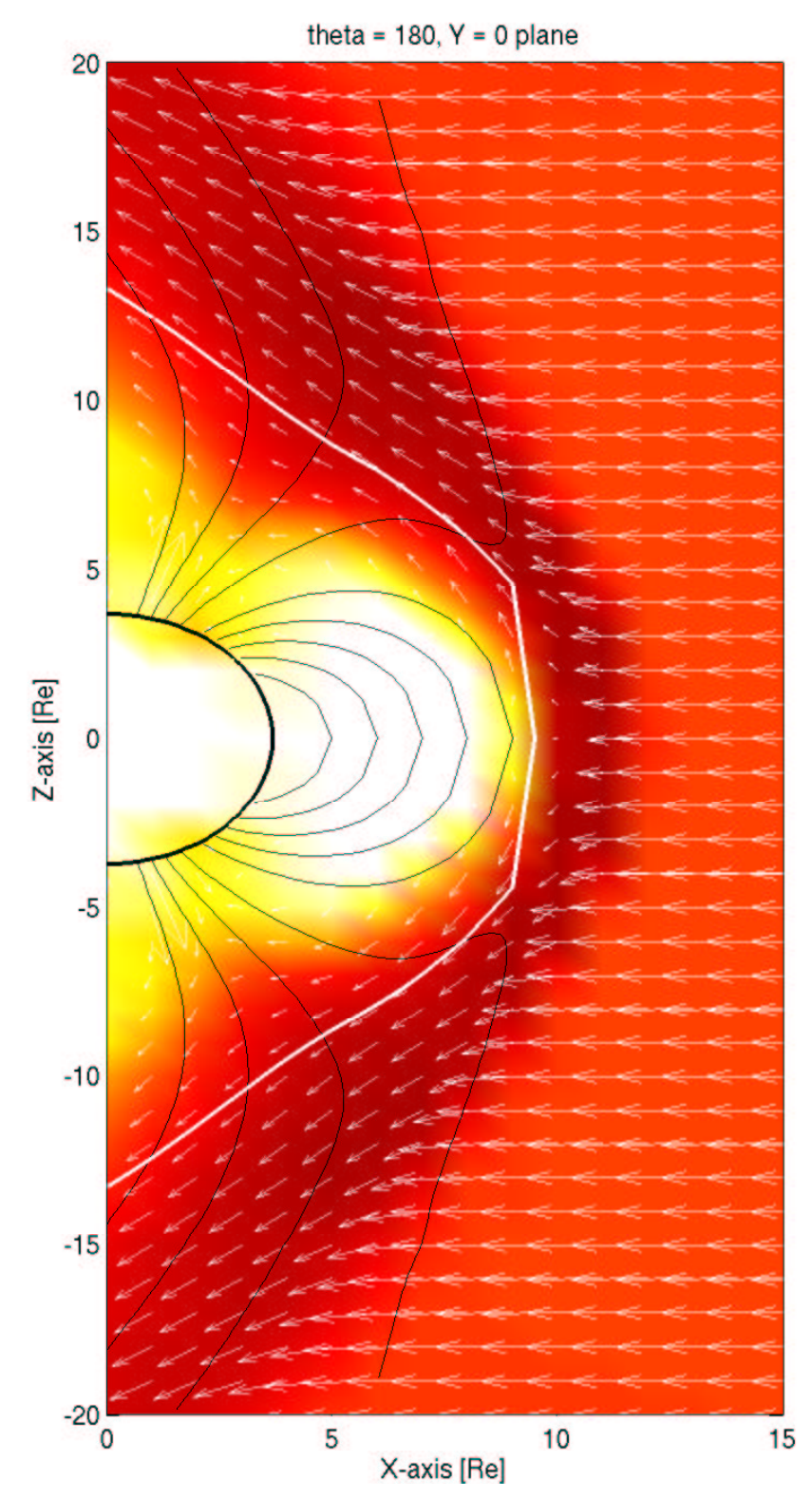

Fig. 4. GUMICS-4 plasma flow pattern (white arrows) at the vicinity of the magnetopause (white thick line) in $X Z$ plane in Run \#1 during due south IMF. Color coding shows the logarithm of density, where white is $3 \# / \mathrm{cm}^{3}$, and dark red $100 \# / \mathrm{cm}^{3}$. Magnetic field lines (black) end to $3.7 R_{E}$ inner shell of MHD domain.

In Fig. 2f, the energy transfer has moved to dusk (dawn) high latitudes at Northern (Southern) Hemisphere. This is in accordance with Fig. 3b, which shows that the field lines opened by low-latitude reconnection convect through dusk (dawn) high latitudes in the Northern (Southern) Hemisphere, allowing Poynting flux focussing and energy transfer in these sectors. The energy transfer is larger during $\theta=300^{\circ}$ (Fig. 2f) than $\theta=60^{\circ}$ (Fig. 2b), although the instantaneous exterior conditions during these two time instants are exactly similar (except for the sign of IMF $y$ component). As the reconnection line in Fig. $2 \mathrm{f}$ has rotated to dawn (dusk) sector in Northern (Southern) Hemisphere, the energy input from these sectors has ceased. Therefore the larger energy input in Fig. $2 \mathrm{f}$ is due to more enhanced convection in the primary energy transfer sectors perpendicular to the reconnection line. The more enhanced convection in these sectors may be explained by the orientation of the reconnection line, which is located at slightly lower latitudes (about from $320^{\circ}$ to $140^{\circ}$, whereas in Fig. $2 \mathrm{~b}$ it is aligned from $30^{\circ}$ to $210^{\circ}$ ). Furthermore, the low latitude reconnection has not ceased and the reconnection is still taking place at the very nose of the magnetopause (unlike in Fig. 2b). Hence, comparison of Figs. 2b and $2 \mathrm{f}$ suggests that reconnection follows the IMF rotation with a delay, explaining the larger energy input in Fig. 2f. Overall, the GUMICS-4 magnetopause reconnection seems to be consistent with the component reconnection model.

\subsection{Mass transfer at the magnetopause}

Since the method for energy transfer (Palmroth et al., 2003) determines the magnetopause as the surface encompassed by the solar wind stream lines, it seems meaningless to replace $\boldsymbol{K}$ in Eq. (1) by $\rho \boldsymbol{v}$ because the magnetopause given by the method should be parallel to magnetosheath velocity field. However, as Fig. 4 indicates, the subsolar region and especially the region where the magnetosheath flow is accelerated and diverted to the different hemispheres are locations on the surface, where the surface normal is not perpendicular to the magnetosheath velocity field. While Fig. 4 is for due southward IMF, the tailward acceleration is always larger in the perpendicular direction than it is parallel to the reconnection line (not shown). Furthermore, a reconnection line includes flow towards and away from the reconnection region. Hence, we hypothesize a priori that replacing $\boldsymbol{K}$ by $\rho \boldsymbol{v}$ in Eq. (1) will characterize mass transfer at locations, where also reconnection is taking place. Notice that the method does not make a distinction about which process transfers the mass, other than it will occur near the reconnection region. Hence, diffusion through the reconnection region might also play a part in the results.

Figure 5a presents the total integrated mass through the magnetopause in the four runs specified in Table 1 as a function of clock angle (and time); vertical dashed lines refer to Fig. 6. Figure $5 \mathrm{~b}$ is the total transferred mass through the dayside portion of the magnetopause. Negative (positive) values indicate that the net transfer is into (out of) the magnetosphere. For other runs except for Run \#3 (large IMF and small dynamic pressure) the net effect is mass inflow for all clock angles. Run \#3 indicates net mass outflow for clock angles between $330^{\circ}$ and $30^{\circ}$. A closer inspection of the simulation results indicate that this outflow takes place tailward of the dawn-dusk terminator, while the dayside still shows mass inflow (Fig. 5b). Larger mass inflow occurs in runs with larger dynamic pressure than for runs with smaller 


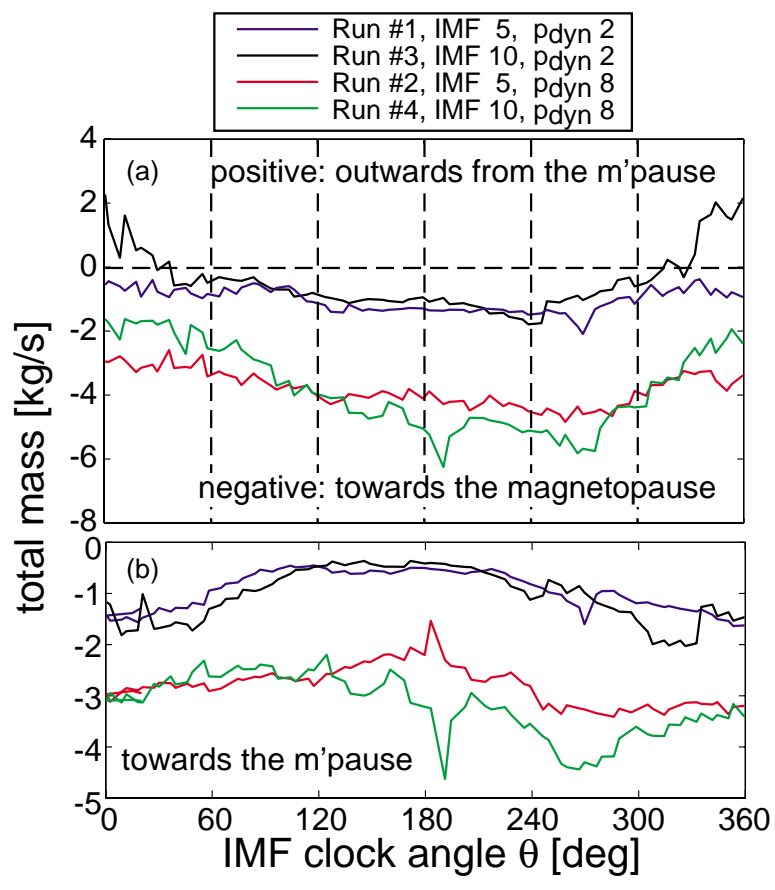

Fig. 5. Total transferred mass through (a) the entire magnetopause between subsolar position and $-30 R_{E}$, and (b) sunward of the terminator as a function of clock angle (and time) in the four runs.

dynamic pressures both on the dayside and on the entire surface. The net inflow in all runs is relatively steady as a function of the clock angle through the entire surface, but the dayside shows generally larger (smaller) mass inflow during northward (southward) IMF.

In Fig. 6, we present the mass transfer distribution for the entire magnetopause from the nose to $-30 R_{E}$ as a function of the IMF clock angle. The format of Fig. 6 is the same as in Fig. 2: the distribution is presented for azimuthal directions shown at the outer circle, and each sector shows the net mass transfer (normalized to $1 \mathrm{~kg} \mathrm{~s}^{-1}$ at the outer circle). Blue (red) color indicates net mass inflow (outflow), and black circles are where FFJ condition holds (again, nose of the magnetopause is at the center of the panel and dawndusk terminator at the outer circle). The red arrow is the clock angle direction, and the times shown are indicated as vertical dashed lines in Fig. 5 .

Panels (a-f) of Fig. 6 show that the mass inflow occurs aligned to the reconnection line for all clock angles. This also proves correct our hypothesis that the mass inflow through the surface determined by stream lines occurs where also reconnection is taking place. Mass outflows are in action in the low-latitude sectors for clock angles between $300^{\circ}$ and $60^{\circ}$. Although Fig. 6 is an integration through the entire length of the magnetopause from the nose to $X=-30 R_{E}$, the mass inflow occurs in the dayside, while the mass outflow occurs in the nightside. We also note that the mass inflow is larger
Run \#1: small IMF, small pdyn: integrated sectorwise from nose to -30 RE
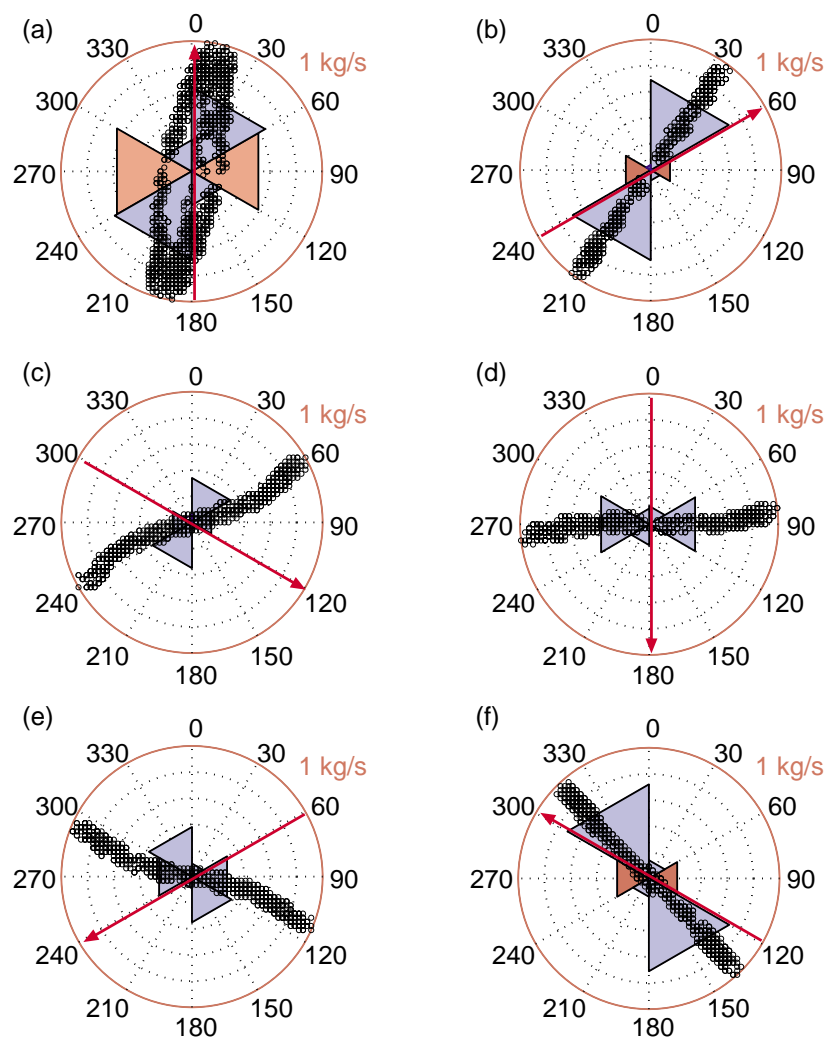

Fig. 6. (a-f) Instantaneous distributions of azimuthal magnetopause mass transfer at angles indicated by dashed vertical lines in Fig. 5, which presents the time evolution of net transferred mass for all runs. Blue (red) color indicates inward (outward) mass at magnetopause in sectors shown outside the outer circle. The sectors scale from $0 \mathrm{~kg} \mathrm{~s}^{-1}$ at the centre to $1 \mathrm{~kg} \mathrm{~s}^{-1}$ at the outer circle. The IMF clock angle direction is indicated by a red arrow, while black circles show the locations where the "four-field junction" condition holds; i.e., where reconnection is likely to occur. Notice that the dayside black circles in panel (a) indicate sunward convection, not reconnection, which occurs behind the cusps (as deduced from the annihilation of magnetic energy). Although the figure is for Run \#1, the other runs behave qualitatively similarly.

for northward than for southward IMF, in accordance with Fig. 5b. A closer inspection of the simulation results reveals that the larger mass inflow during lobe reconnection conditions is due to plasma capture by sunward convecting field lines that close on the dayside. However, simultaneous inflow and outflow during northward IMF compensate and lead to the flat total integral in Fig. 5a. Although Fig. 6 is for Run \#1, the other runs behave qualitatively similarly (inflow from dayside along the reconnection line, outflow during northward IMF from the low-latitude tail). 


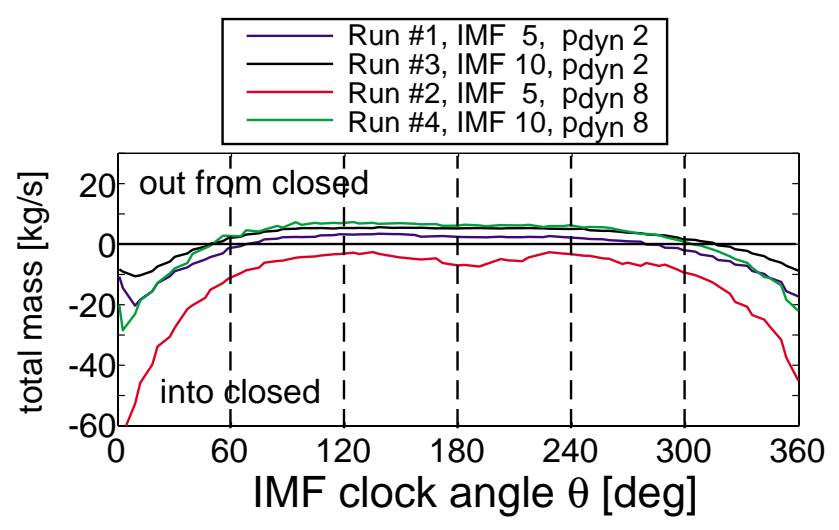

Fig. 7. Net transferred mass to dayside closed field as a function of IMF clock angle (and time) for all runs.

\subsection{Mass transfer to the closed field}

To complete our analysis of mass inflow to the magnetosphere, we examine the preferential conditions for mass inflow to closed field as it is not self-evident that the mass transferred inside the magnetopause is readily available for the system dynamics. Hence, in Fig. 7 we present the net mass flux through the surface formed by the last closed field lines as a function of the clock angle in the four runs; negative (positive) values indicate mass flux to (out from) the closed field (for the method of identifying the surface, see Laitinen et al. (2006)). The computation is for the dayside portion of the surface only. Figure 7 indicates that the net effect of mass transfer is towards the closed field during northward IMF, and outwards of the closed field during southward IMF in other runs except for Run \#2 (small IMF and large pressure), where the net effect is towards the closed field at all clock angles. However, in all runs the largest flux to the closed field takes place during northward IMF, while for southward IMF the mass flux to closed field decreases and even becomes flux towards the open field. Note that the magnitude of the mass flux is larger than through the magnetopause as the surface is different and not necessarily parallel to the magnetosheath velocity field. Hence there is a larger $\rho \boldsymbol{v} \cdot \hat{\boldsymbol{n}}$ at the surface.

Panels (a-f) of Fig. 8 show the net flux towards the dayside closed field in Run \#1; the format of the figure is similar as in Fig. 6. Again, the mass flux towards the closed field is at maximum during northward IMF as then the sunward convecting field lines opened by lobe reconnection close on the dayside capturing also a large amount of plasma to the closed field. The flux towards the closed field always occurs where reconnection is occurring, indicating that the two different methods (mass flux through magnetopause and to closed field) yield a similar result regardless of the choice of the surface. Outflow from the closed field occurs in sectors perpendicular to the reconnection line, i.e., in sectors where convection is taking place.
In contrast to the magnetopause, where the azimuthal distribution of energy and mass transfer is qualitatively similar in all runs (behaves as in Figs. 2 and 6, respectively; only magnitudes are different), the mass flux to closed field varies according to the solar wind conditions. Panels $(\mathrm{a}-\mathrm{c})$ of Fig. 9 present an overview of the azimuthal distribution for the dayside mass transfer to closed field for Runs \#2, \#3, and \#4, respectively, for the due southward IMF; the format of the figure is similar to Fig. 8d.

Figure 9 shows that the mass inflow component during southward IMF along the reconnection line is present in other runs (Fig. 8d, and Figs. 9a and c) except for during large IMF and small dynamic pressure (Fig. 9b). The mass outflow component in the sectors where convection is taking place is, however, present in all runs. The relative magnitudes of the mass inflow and outflow components in the different runs are different: For small IMF and large pressure (Fig. 9a) the mass inflow component along the reconnection line is larger than the mass outflow components in the convection sectors. For large IMF and large pressure (Fig. 9c), the inflow component along the reconnection line is decreased while the outflow component in the convection sectors is of similar magnitude as for small IMF and large pressure (Fig. 9a). This suggests that the outflow in the convection sectors is controlled by the dynamic pressure (as it remains unchanged from Figs. 9a to c). It also suggests that the inflow component along the reconnection line decreases for increasing IMF; this can also be seen by comparing Fig. 8d with Fig. 9b. In Run \#4 the outflow component in the convection sectors is generally larger than the inflow component along the reconnection line, while the opposite is true for Run \#2. In Run \#1, however, the inflow and outflow components are relatively of the same magnitude throughout the southward IMF (Fig. 8). Table 2 summarizes the changes in the mass inflow (along the reconnection line) and outflow (in the convection sectors) components during southward IMF as a function of IMF and dynamic pressure.

\section{Discussion}

In this paper we have used a global MHD code GUMICS-4 to investigate the energy and mass transfer through the magnetopause and towards the closed magnetic field. We have studied the energy and mass transfer as a response to changing clock angle $\theta$, IMF magnitude, and solar wind dynamic pressure. We find here and in Palmroth et al. (2006) that while the energy transfer follows best the $\sin ^{2}(\theta / 2)$ dependence, there is more energy transfer after large energy input. There is no clear clock angle dependence in the net mass transfer through the magnetopause, but the mass transfer through the dayside magnetopause and towards the closed field occurs favorably for northward IMF. The energy transfer occurs through areas at the magnetopause that are perpendicular to the reconnection line. In contrast, the mass transfer occurs consistently 
Table 2. Changes in mass transfer to closed field in different runs.

\begin{tabular}{lccc}
\hline & inflow (along rec. line) & outflux (conv. sectors) & Figs. to compare \\
\hline Const. $p_{\text {dyn }}$ (small): small IMF $\rightarrow$ large IMF & decrease & $\sim$ same & $8 \mathrm{~d}, 9 \mathrm{~b}$ \\
Const. $p_{\text {dyn }}$ (large): small IMF $\rightarrow$ large IMF & decrease & $\sim$ same & $9 \mathrm{a}, 9 \mathrm{c}$ \\
\hline Const. IMF (small): small $p_{\text {dyn }} \rightarrow$ large $p_{\text {dyn }}$ & increase & increase & $8 \mathrm{~d}, 9 \mathrm{a}$ \\
Const. IMF (large): small $p_{\text {dyn }} \rightarrow$ large $p_{\text {dyn }}$ & increase & increase & $9 \mathrm{~b}, 9 \mathrm{c}$ \\
\hline
\end{tabular}
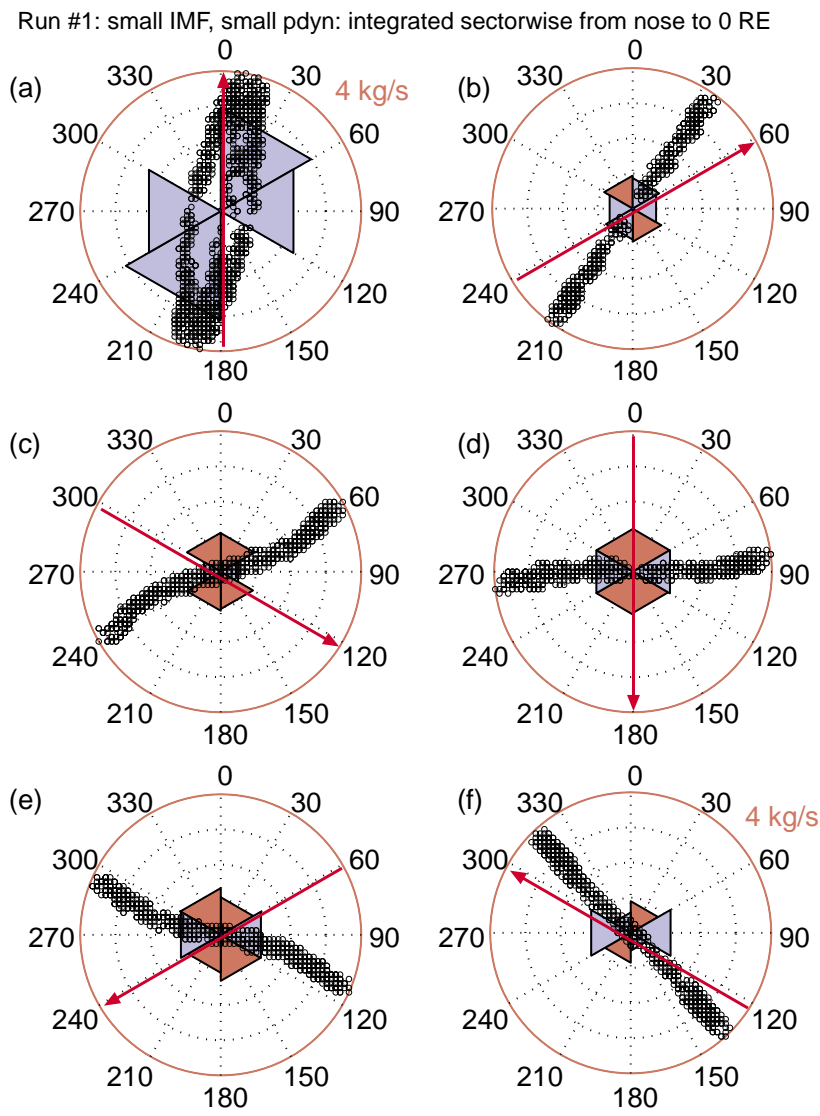

Fig. 8. (a-f) Instantaneous distributions of azimuthal mass transfer to dayside closed field for Run \#1 at angles indicated by dashed vertical lines in Fig. 7. Blue (red) color indicates towards closed (out from closed) field mass transfer in sectors shown outside the outer circle. The sectors scale from $0 \mathrm{~kg} \mathrm{~s}^{-1}$ at the centre to $4 \mathrm{~kg} \mathrm{~s}^{-1}$ at the outer circle. The IMF clock angle direction is indicated by a red arrow, while black circles show the locations where the "fourfield junction" condition holds; i.e., where reconnection is likely to occur. Note that the dayside black circles in panel (a) indicate sunward convection, not reconnection, which occurs behind the cusps (as deduced from the annihilation of magnetic energy).

along the reconnection line, both through the magnetopause and towards the closed field. Both the energy and mass transfer are enhanced in response to increased solar wind dynamic

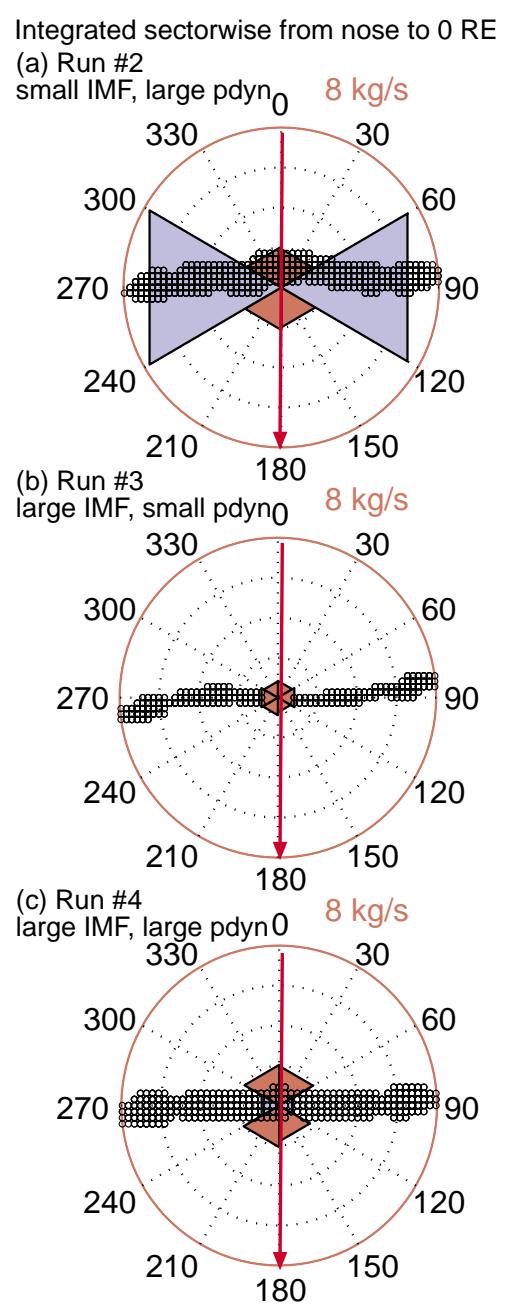

Fig. 9. (a-c) Instantaneous distributions of azimuthal mass transfer to dayside closed field for Run \#2, \#3, and \#4, respectively, for due south IMF. Blue (red) color indicates towards closed (out from closed) field mass transfer in sectors shown outside the outer circle. The sectors scale from $0 \mathrm{~kg} \mathrm{~s}^{-1}$ at the centre to $8 \mathrm{~kg} \mathrm{~s}^{-1}$ at the outer circle. The IMF clock angle direction is indicated by a red arrow, while black circles show the locations where the "four-field junction" condition holds; i.e., where reconnection is likely to occur. 
pressure, while increasing the IMF magnitude does not affect the transfer quantities as much.

Using a predefined simulation box with fixed boundary conditions, Birn et al. (2001) showed that the reconnection rate as measured by the parallel electric field is slower in the MHD formulation as compared to other numerical techniques. However, in a global MHD code, the boundary conditions are self-consistent and follow global dynamics. As shown by Laitinen et al. (2005), the tail reconnection processes half of the incoming magnetopause energy, indicating that the GUMICS-4 tail reconnection is an efficient process and central to the magnetospheric dynamics. However, even if the GUMICS-4 reconnection rate would be too small (of which we have no evidence), the global energy and mass transfer may still correspond to reality at the magnetopause: Energy transfer takes place because open field lines moving tailward form an angle between the magnetosheath velocity field, allowing Poynting flux to focus towards the magnetopause. Hence, the reconnection rate is not a large issue in the global energy transfer, as long as it creates enough open field lines at the magnetopause. This is clearly the case in GUMICS-4, otherwise the Poynting flux would be zero at the magnetopause. On the other hand, the mass transfer through the magnetopause only needs a place where the magnetosheath velocity field is not parallel to the surface, which occurs near the reconnection region. Even with a small reconnection rate one can still have a roughly right location for the reconnection line, and the results may still be quantitative taken that the magnetosheath parameters are correct. Hence, we believe that our results on the energy and mass transfer may still be quantitative and reflect the global properties at the magnetopause.

\subsection{Energy transfer}

Pudovkin et al. (1986) suggested that the Poynting flux through the magnetopause is proportional to $\sin ^{2}(\theta-\phi)$, where $\phi$ is the angle at which the stagnation line lies at the magnetopause. The results in this paper are in agreement with Sonnerup (1974) and Kan and Lee (1979), and imply that $\phi \approx \theta / 2$. However, according to the results shown here, energy transfer through the magnetopause is not only a function of $\theta$, but it remains enhanced after large energy input (the "hysteresis" effect discussed in Sect. 3.1 and in Palmroth et al. (2006)). The simulation results indicate that 1 ) the subsolar reconnection remains active after it has started (compare e.g., Figs. $2 b$ and f), 2) the reconnection line follows the IMF rotation with a delay, and 3) the previously active convection maintains the Poynting flux focussing for a while in some areas of the magnetopause, even though the reconnection line orientation would already accommodate energy transfer in some other area of the magnetopause. If observationally verified, the hysteretic behavior of reconnection may lead to important consequences in magnetospheric physics, as some of the delays associated with the system energetics may already arise from processes taking place at the magnetopause (see also Pulkkinen et al., 2006). In the opposite case we learn more of the capabilities of global MHD to model the system behavior.

Recently, Klimas et al. (2005) criticize the dynamics in the global MHD codes for being too directly driven by the solar wind and IMF driver. They assert that the "substorm in the magnetotail is hysteretic: Magnetic flux is added to the tail until the threshold of a still-undetermined instability in the tail is reached at which point unloading begins with the onset of a substorm". They suggest that an instability is triggered when a critical current density in the tail is reached, while quenching of the instability occurs when the current density decreases to below another critical current density, smaller than what was required for the instability triggering. Using such formulation leads to a loading-unloading cycle in a driven current sheet even under continuous, steady driving. However, it is interesting to note that no such implementation of critical current density thresholds is needed at the GUMICS-4 magnetopause to get the hysteretic behavior of the dayside reconnection line location and energy transfer efficiency.

It is often implicitly assumed that the energy transfer would occur near the reconnection line (e.g., Pudovkin et al., 1986). Furthermore, the parameter $l_{0}$ in $\epsilon$ (Akasofu, 1981) that was originally used to scale the input to equal the output, has been interpreted as the radius of the energy transfer area. This justifies the representation of $\epsilon$ as the "Poynting flux through an area" $\left(\epsilon \propto 4 \pi l_{0}^{2} \cdot\left(1 / \mu_{0}\right) v B^{2}\right)$. The results shown in here and in Palmroth et al. (2003) indicate that the area through which the energy transfer takes place is neither close to the reconnection line nor directly scalable by the magnetopause area. This implies that the energy transfer proxies need to be further refined to arrive at a fully quantitative description of the system input. According to the simulation results, the energy transfer takes place at locations where the magnetosheath velocity field and the open field lines dragging tailward form a finite angle with each other, allowing the Poynting vector to have a component towards the magnetopause. Hence, the electromagnetic energy transfer accommodates a geometrical demand arising from tailward moving field lines and magnetosheath velocity field.

Due to the vast area of the magnetopause, the spatial variation of energy transfer shown in here and in Palmroth et al. (2003) has never been observationally verified. However, there are some studies that support the simulation results. For example, the dayside auroral activity is thought to be a manifestation of direct energy transfer, as the so-called "afternoon hot spot" responds to variations in convection, which in turn responds to variations in the solar wind (Fillingim et al., 2005). Using simultaneous observations from both hemispheres, Fillingim et al. (2005) showed that the afternoon hot spot is active in the Southern (Northern) Hemisphere during positive (negative) IMF $y$ and southward IMF. Figure 2 indicates that this is in agreement with the simulation results: the 
afternoon sector shows most enhanced energy transfer in the Southern Hemisphere during positive IMF $y$ and southward IMF (Fig. 2c). On the other hand, the afternoon sector in the Northern Hemisphere shows most enhanced energy transfer during negative IMF $y$ and southward IMF (Fig. 2e).

\subsection{Mass transfer}

First, we remind that the method for detecting the magnetopause through which the transfer quantities are computed is based on solar wind stream lines. As there are always flows toward and away from the reconnection line, the reconnection region will have a component of $\rho \boldsymbol{v}$ towards the magnetopause. Thus, it is not surprising that the mass inflow through the magnetopause from the method occurs along the reconnection line rotating with the IMF clock angle. However, the surface contained by the last closed field lines is determined by a completely independent method (Laitinen et al., 2006). As the mass influx from the two methods are qualitatively in agreement, we may assert that both methods characterize the physics in the simulation and are not dependent on the methods. The magnetopause of GUMICS-4 is statistically in accordance with the magnetopause of Shue et al. (1998) (Palmroth et al., 2001), and that the magnetosheath parameters given by the global MHD simulations are generally in agreement with observations (e.g., Koval et al., 2006). Furthermore, the GUMICS-4 magnetopause reconnection is consistent with component reconnection model, which is in agreement with several observational papers (e.g., Phan et al., 2006). Hence our results on the mass transfer may well characterize the mass transfer through the observational magnetopause.

The simulation results indicate that the net mass transfer at the reconnection line is a few kilograms per second. This amount for mass inflow through the entire magnetopause corresponds quantitatively with previous assessments: Sibeck et al. (1999) estimate that $3 \%$ of the incident $10^{29}$ ions per second enters the magnetopause, yielding $\sim 5 \mathrm{~kg} \mathrm{~s}^{-1}$ total mass entry through the entire magnetopause (assuming that the majority of incident ions are protons). However, Fig. 5 indicates that this amount can enter solely at the reconnection location.

According to GUMICS-4, there is more mass transfer during large solar wind dynamic pressures, regardless of the magnitude of the IMF. The large dynamic pressure was implemented by increasing both the velocity and density in the solar wind, in order to avoid possible problems arising from unrealistically high values in one or the other. Hence, the $\rho v$ in the magnetosheath is larger in the runs having a large dynamic pressure. The amount of mass crossing the magnetopause should be related to the amount of mass in the plasma sheet, because the system is in pressure balance in the quasistationary sense (plasma sheet with lobes and lobes with magnetosheath). Using the Medium Energy Neutral Atom (MENA) imager onboard the Image satellite, McComas et al.
(2002) found that the high densities both in the plasma sheet and at geosynchronous orbit are associated with high density in the solar wind, regardless of the IMF orientation. Furthermore, Borovsky et al. (1998) show that the plasma sheet density is positively correlated with the solar wind density, again without grouping the observations according to the IMF orientation. Notice that the transfer rate does not have to depend on the solar wind density, as denser plasma sheet would result from a denser solar wind also for a constant transfer rate.

Our results show more mass transfer towards the closed field during northward IMF. This is mainly due to lobe reconnection occurring simultaneously in both hemispheres, creating closed field lines at the dayside and in the process capturing plasma to the closed field. As many observational studies show, the plasma sheet becomes denser and cooler for prolonged periods of northward IMF (e.g., Wing and Newell, 2002; Tsyganenko and Mukai, 2003), indicating that mass transfer does occur for northward IMF. Consistent with observations (Wing and Newell, 2002), the GUMICS4 plasma sheet is hot and tenuous during southward IMF and cool and dense during northward IMF. This is shown in Fig. 10, where a cut through the magnetosphere in the $Y Z$ plane at $X=-8 R_{E}$, within the GUMICS-4 plasma sheet, is presented. The first (second) row shows the density and temperature for northward (southward) IMF. Hence, we suggest that during northward IMF plasma transfers into the dayside closed field, and later this plasma moves into the plasma sheet through convection, as Li et al. (2005) suggests. During southward IMF there is not enough time to fill the plasma sheet as the transferred mass is readily heated and accelerated towards the Earth and tailward at the tail reconnection region. Hence, during southward IMF there is an active emptying mechanism that may in some occasions push the plasma also out from the closed field region. During northward IMF there is nothing to transport mass out from the plasma sheet, and hence after a while the plasma sheet gets denser and cooler, consistent with observational studies.

The simulation results indicate that the energy and mass inflow through the magnetopause are qualitatively similar in the different runs, but the mass transfer towards the closed field varies qualitatively in the different runs. Figure 9 and Table 2 show that there are two components in the closed field mass transfer, whose relative impact depends on the combination of solar wind dynamic pressure and the IMF magnitude. The inflow component along the reconnection line increases with increasing pressure, but decreases with increasing IMF magnitude. The outflow component in the convection sectors does not depend on the IMF magnitude, but increases with increasing solar wind dynamic pressure. The pressure dependence may be explained in the following way: First, high pressures are required to bring the magnetosheath field lines efficiently to the magnetopause, where they are reconnected. Second, the opened field lines are also efficiently carried away by the large magnetosheath $\rho \boldsymbol{v}$ towards the tail. 

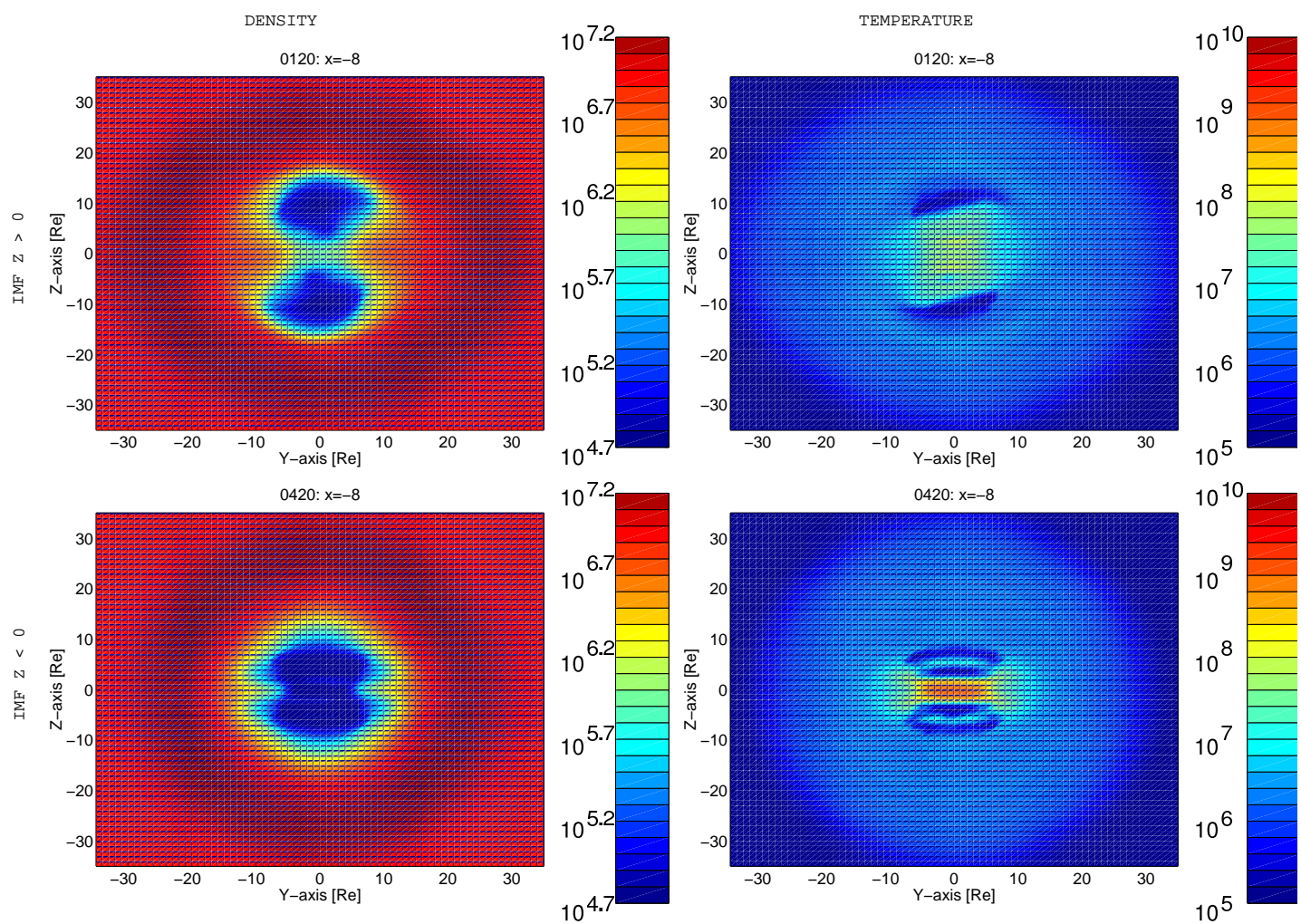

Fig. 10. Logarithm of density (left) and temperature (right) in the GUMICS-4 simulation at $X=-8 R_{E}$, within the simulation plasma sheet. First row shows the parameters for northward IMF (locally at $-8 R_{E}$ ), while the second row is for southward IMF. The color bars range from $10^{4.7}$ to $10^{7.2} \# / \mathrm{m}^{3}$ for the density, and from $10^{5}$ to $10^{10} \mathrm{~K}$ for the temperature. The figure is from Run \#1.

However, if the solar wind dynamic pressure is constant, and only the IMF magnitude increases, the inflow component towards the closed field along the reconnection line decreases (Table 2). This means that the mass outflow from the closed field is enhanced. Phan et al. (2005) report of an event during which low latitude reconnection appears to be in process of eroding a pre-existing dayside low-latitude boundary layer (LLBL); which, during the event (northward IMF and substantial IMF $y$ ), should lie on closed field lines (Phan et al., 1997). Although the event of Phan et al. (2005) occurs during northward IMF, it has an active low-latitude reconnection component, as in Fig. 9. Likewise, although Phan et al. (2005) do not consider an increasing IMF magnitude, they show that during low-latitude reconnection there is mass outflow from the closed field lines. Hence, the following assumptions together explain our results: 1) low-latitude reconnection erodes mass out from the closed field (Phan et al., 2005), and that 2) larger IMF increases this effect (as suggested by Fig. 7), while the effect may be absent during small IMF and large pressure (see Fig. 7). We suggest that the mass erosion out from the closed field during southward IMF may be related to the active reconnection process emptying the plasma sheet.

\section{Conclusion and summary}

In conclusion, we find that a global MHD codes can be used in quantifying and characterizing the energy and mass flow through the magnetopause and towards the closed field, as many of the simulation results are consistent with observational results. According to GUMICS-4, the mass and energy transfer at the magnetopause are different both in spatial characteristics and in response to changes in the solar wind parameters. The findings of this paper are summarized as follows:

1. Energy transfer follows best the $\sin ^{2}(\theta / 2)$ dependence, but there is more energy transfer after large energy input, and that the reconnection line follows the IMF rotation with a delay.

2. Energy transfer occurs through areas at the magnetopause that are perpendicular to the reconnection line. Energy transfer is due to Poynting flux focussing, arising from a geometrical demand between the tailward moving field lines and magnetosheath velocity field.

3. Mass transfer occurs along the reconnection line, both through the magnetopause and towards the closed field. 
The magnitude of mass transfer through the magnetopause is a few kilograms per second.

4. There is no clear clock angle dependence in the net mass transfer through the magnetopause, but the mass transfer through the dayside magnetopause and towards the closed field occurs favorably for northward IMF. During such conditions the sunward convecting field lines opened by lobe reconnection close on the dayside capturing also a large amount of plasma to the closed field.

5. Both the energy and mass transfer are enhanced in response to increased solar wind dynamic pressure, while increasing the IMF magnitude does not affect the transfer quantities as much.

Acknowledgements. The work of M. Palmroth is supported by the Academy of Finland. The work of T. V. Laitinen was supported by the Magnus Ehrnrooth Foundation. GUMICS-4 code is developed by P. Janhunen. We thank P. Janhunen and H. E. J. Koskinen for fruitful discussions on the energy and mass transfer.

Topical Editor I. A. Daglis thanks two referees for their help in evaluating this paper.

\section{References}

Akasofu, S.-I.: Energy coupling between the solar wind and the magnetosphere, Space Sci. Rev., 28, 121-190, 1981.

Birn, J., Drake, J. F., Shay, M. A., Rogers, B. N., Denton, R. E., Hesse, M., Kuznetsova, M., Ma, Z. W., Bhattacharjee, A., Otto, A., and Pritchett, P. L.: Geospace Environmental Modeling (GEM) Magnetic Reconnection Challenge, J. Geophys. Res., 106, 3715-3719, 2001.

Borovsky, J. E., Thomsen, M. F., and Elphic, R. C.: The driving of the plasma sheet by the solar wind, J. Geophys. Res., 103(A8), 17 617-17 640, 1998.

Burton, R. K., McPherron, R. L., and Russell, C. T.: An empirical relationship between interplanetary conditions and Dst, J. Geophys. Res., 80, 4204-4214, 1975.

Cowley, S. W. H., Morelli, J. P., and Lockwood, M.: Dependence of convective flows and particle precipitation in the high-latitude dayside ionosphere on the $\mathrm{X}$ and $\mathrm{Y}$ components of the interplanetary magnetic field, J. Geophys. Res., 96, 5557-5564, 1991.

Dungey, J. W.: Interplanetary magnetic field and the auroral zones, Phys. Rev. Lett., 6, 47-48, 1961.

Fillingim, M. O., Parks, G. K., Frey, H. U., Immel, T. J., and Mende, S. B.: Hemispheric asymmetry of the afternoon electron aurora, Geophys. Res. Lett., 32, L03113, doi:10.1029/2004GL021635, 2005.

Hasegawa, H., Fujimoto, M., Phan, T.-D., Reme, H., Balogh, A., Dunlop, M. W., Hashimoto, C., and TanDokoro, R.: Transport of solar wind into Earth's magnetosphere through rolled-up KelvinHelmholtz vortices, Nature, 430, 755-757, 2004.

Hubert, B., Palmroth, M., Laitinen, T. V., Janhunen, P., Milan, S. E., Grocott, A., Cowley, S. W. H., Pulkkinen, T., and Gérard, J.-C.: Compression of the Earth's magnetotail by interplanetary shocks directly drives transient magnetic flux closure, Geophys. Res. Lett., 33, L10105, doi:10.1029/2006GL026008, 2006.
Janhunen, P.: GUMICS-3: A global ionosphere-magnetosphere coupling simulation with high ionospheric resolution, in: Proceedings of Environmental Modelling for Space-Based Applications, Sept. 18-20 1996, Eur. Space Agency Spec. Publ., ESA SP-392, pp. 233-239, 1996.

Johnson, J. R. and Cheng, C. Z.: Kinetic Alfvén waves and plasma transport at the magnetopause, Geophys. Res. Lett., 24, 14231426, 1997.

Kan, J. R. and Lee, L. C.: Energy coupling function and solar windmagnetosphere dynamo, Geophys. Res. Lett., 6, 577-581, 1979.

Klimas, A. J., Uritsky, V. M., Vassiliadis, D., and Baker, D. N.: A mechanism for the loading-unloading substorm cycle missing in MHD global magnetospheric simulation models, Geophys. Res. Lett., 32(14), L14108, doi:10.1029/2005GL022916, 2005.

Koval, A., Šafránková, J., Němeček, Z., Samsonov, A. A., Přech, L., Richardson, J. D., and Hayosh, M.: Interplanetary shock in the magnetosheath: Comparison of experimental data with MHD modeling, Geophys. Res. Lett., 33, L11102, doi:10.1029/2006GL025707, 2006.

Laitinen, T. V., Pulkkinen, T. I., Palmroth, M., Janhunen, P., and Koskinen, H. E. J.: The magnetotail reconnection region in a global MHD simulation, Ann. Geophys., 23, 3753-3764, 2005, http://www.ann-geophys.net/23/3753/2005/.

Laitinen, T. V., Janhunen, P., Pulkkinen, T. I., Palmroth, M., and Koskinen, H. E. J.: On the characterization of magnetic reconnection in global MHD simulations, Ann. Geophys., in press, 2006.

Li, W., Raeder, J., Dorelli, J., Øieroset, M., and Phan, T. D.: Plasma sheet formation during long period of northward IMF, Geophys. Res. Lett., 32, L12S08, doi:10.1029/2004GL021524, 2005.

Lu, G., Onsager, T. G., Le, G., and Russell, C. T.: Ion injections and magnetic field oscillations near the high-latitude magnetopause associated with solar wind dynamic pressure enhancement, J. Geophys. Res., 109, A06208, doi:10.1029/2003JA010297, 2004.

Luhmann, J. G., Walker, R. J., Russell, C. T., Crooker, N. U., Spreiter, J. R., and Stahara, S. S.: Patterns of potential magnetic field merging sites on the dayside magnetopause, J. Geophys. Res., 89, 1739-1742, 1984.

McComas, D. J., Valek, P., Burch, J. L., Pollock, C. J., Skoug, R. M., and Thomsen, M. F.: Filling and emptying of the plasma sheet: Remote observations with $1-70 \mathrm{keV}$ energetic neutral atoms, Geophys. Res. Lett., 29(22), 2079, doi:10.1029/2002GL016153, 2002.

Nykyri, K. and Otto, A.: Plasma transport at the magnetospheric boundary due to reconnection in Kelvin-Helmholtz vortices, Geophys. Res. Lett., 28, 3565-3568, 2001.

Øieroset, M., Phan, T. D., and Fujimoto, M.: Wind observations of asymmetric magnetic reconnection in the distant magnetotail, Geophys. Res. Lett., 31, L12801, doi:10.1029/2004GL019958, 2004.

Palmroth, M., Janhunen, P., Pulkkinen, T. I., and Peterson, W. K.: Cusp and magnetopause locations in global MHD simulation, J. Geophys. Res., 106, 29 435-29450, 2001.

Palmroth, M., Pulkkinen, T. I., Janhunen, P., and Wu, C.-C.: Stormtime energy transfer in global MHD simulation, J. Geophys. Res., 108(A1), 1048, doi:10.1029/2002JA009446, 2003.

Palmroth, M., Janhunen, P., and Pulkkinen, T. I.: Hysteresis in solar wind power input to the magnetosphere, Geophys. Res. Lett., 33, L03107, doi:10.1029/2005GL025188, 2006. 
Parker, E. N.: Sweets mechanism for merging magnetic fields in conducting fluids, J. Geophys. Res., 62, 509-520, 1957.

Phan, T. D., Larson, D., McFadden, J., Lin, R. P., Carlson, C., Moyer, M., Paularena, K. I., McCarthy, M., Parks, G. K., Rème, H., Sanderson, T. R., and Lepping, R. P.: Low-latitude dusk flank magnetosheath, magnetopause, and boundary layer for low magnetic shear: Wind observations, J. Geophys. Res., 102, 1988319896, 1997.

Phan, T.-D., Oieroset, M., and Fujimoto, M.: Reconnection at the dayside low latitude magnetopause and its nonrole in low-latitude boundary layer formation during northward interplanetary magnetic field, Geophys. Res. Lett., 32, L17101, doi:10.1029/2005GL023355, 2005.

Phan, T. D., Hasegawa, H., Fujimoto, M., Oieroset, M., Mukai, T., Lin, R. P., and Paterson W.: Simultaneous Geotail and Wind observations of reconnection at the subsolar and tail flank magnetopause, Geophys. Res. Lett., 33, L09104, doi:10.1029/2006GL025756, 2006.

Pudovkin, M. I., Semenov, V. S., Heyn,, M. F., and Biernat, H. K.: Implications of the stagnation line model for energy input through the dayside magnetopause, Geophys. Res. Lett., 13, 213-216, 1986.

Pulkkinen, T. I., Palmroth, M., Tanskanen, E. I., Janhunen, P., Koskinen, H. E. J., and Laitinen, T. V.: New interpretation of magnetospheric energy circulation, Geophys. Res. Lett., 33, L07101, doi:10.1029/2005GL025457, 2006.

Shepherd, S. G., Greenwald, R. A., and Ruohoniemi, J. M.: Cross polar cap potentials measured with Super Dual Auroral Radar Network during quasi-steady solar wind and interplanetary magnetic field conditions, J. Geophys. Res., 107(A7), 1094, doi:10.1029/2001JA000152, 2002.
Shue, J.-H., Song, P., Russell, C. T., Steinberg, J. T., Chao, J. K., Zastenker, G., Vaisberg, O. L., Kokubun, S., Singer, H. J., Detman, T. R., and Kawano, H.: Magnetopause location under extreme solar wind conditions, J. Geophys. Res., 103, 17 691-17 700, 1998.

Sibeck, D. G., Paschmann, G., Treumann, R. A., Fuselier, S. A., Lennartsson, W., Lockwood, M., Lundin, R., Ogilvie, K., W., Onsager, T., G., Phan, T.-D., Roth, M., Scholer, M., Sckopke, N., Stasiewicz, K., and Yamauchi, M.: Plasma transfer processes at the magnetopause, in: Magnetospheric Plama sources and losses, edited by: Hultqvist, B., Øieroset, M., Paschmann, G., and Treumann, R., Kluwer Academic Publishers, the Netherlands, pp. 207-285, 1999.

Sonnerup, B. U. Ö.: Magnetopause reconnection rate, J. Geophys. Res., 79, 1546-1549, 1974.

Sweet, P. A.: The neutral point theory of solar flares, in: Electromagnetic Phenomena in Cosmical Physics, IAU Symp. 6 (Cambridge Univ. Press, London), 123-134, 1958.

Thomsen, M. F., Borovsky, J. E., Skoug, R. M., and Smith, C. W.: Delivery of cold, dense plasma sheet material into the near-Earth region, J. Geophys. Res., 108(A4), 1151, doi:10.1029/2002JA009544, 2003.

Tsyganenko, N. A. and Mukai, T.: Tail plasma sheet models derived from Geotail particle data, J. Geophys. Res., 108(A3), 1136, doi:10.1029/2002JA009707, 2003.

Wing, S. and Newell, P. T.: 2D plasma sheet ion density and temperature profiles for northward and southward IMF, Geophys. Res. Lett., 29(9), 1307, doi:10.1029/2001GL013950, 2002. 